\title{
Polyoxometalate based Poly(ionic liquid) as a Precursor for Superhydrophobic Magnetic Carbon Composite Catalysts toward Aerobic Oxidative Desulfurization
}

Wei Jiang, ${ }^{\mathrm{a}}$ Jin Xiao, ${ }^{\mathrm{b}}$ Lei Dong, ${ }^{\mathrm{b}}$ Chao Wang, ${ }^{\mathrm{b}}$ Hongping Li, ${ }^{\mathrm{a}}$ Yaping Luo, ${ }^{\mathrm{b}}$ Wenshuai Zhu, ${ }^{b^{*}}$ Huaming $\mathrm{Li}^{\mathrm{a}}$

anstitute for Energy Research, 301 Xuefu Road, Zhenjiang 212013, P. R. China.

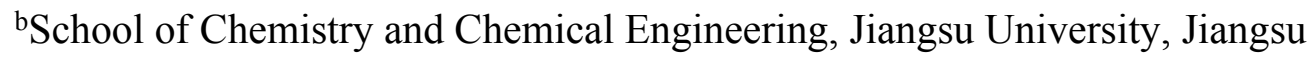

University, 301 Xuefu Road, Zhenjiang 212013, P. R. China.

E-mail: zhuws@ujs.edu.cn; lihm@ujs.edu.cn

The supporting information consists of 4 pages, and 4 figures. 


\section{Instruments}

Fourier transform-infrared (FT-IR) spectra of samples were recorded with a Nicolet FT-IR spectrophotometer (Nexus 470, Thermo Electron Corporation) using $\mathrm{KBr}$ pellets at room temperature. Raman spectra were conducted using a DXR Raman microscope with a $532 \mathrm{~nm}$ laser source. Powder X-ray diffraction (XRD) analysis of samples in the $2 \theta$ range from $10^{\circ}$ to $80^{\circ}$ were performed on Bruker D8 diffractometer with high-intensity $\mathrm{Cu} \mathrm{Ka}(\lambda=1.54 \AA)$. The samples were dispersed in ethanol and dropped into a $\mathrm{Cu}$ grip to carry out a transmission electron microscopy (TEM) experiment with a JEOL-JEM-2010 (JEOL, Japan) operated at $200 \mathrm{kV}$. The $\mathrm{N}_{2}$ adsorptiondesorption isotherms at $77 \mathrm{~K}$ were carried out using a TriStar II 3020 analyzer. The specific surface areas were calculated using the Brunauer - Emmett - Teller (BET) method. The magnetic properties were measured by a superconducting quantum interference device (SQUID) at $300 \mathrm{~K}$. X-ray photoelectron spectroscopy (XPS) was performed using a Thermo ESCALAB 250Xi spectrometer with $\mathrm{Mg} \mathrm{K} \alpha$ radiation. Shimadzu GC-2010 Plus, Rtx- 5 column, $30 \mathrm{~m}$ long $\times 0.25 \mathrm{~mm}$ inner diameter (id) $\times 0.25$ um film thickness; injector port temperature, $250^{\circ} \mathrm{C}$; detector temperature, $300^{\circ} \mathrm{C}$; oven temperature, starting at $100^{\circ} \mathrm{C}$ and rising to $200^{\circ} \mathrm{C}$ at $15^{\circ} \mathrm{C} / \mathrm{min}$ for DBT and $\mathrm{BT}$, starting at $100^{\circ} \mathrm{C}$ and rising to $160^{\circ} \mathrm{C}$ at $20^{\circ} \mathrm{C} / \mathrm{min}$ and then rising to $230^{\circ} \mathrm{C}$ at $25^{\circ} \mathrm{C} / \mathrm{min}$ for $4,6-\mathrm{DMDBT}$.

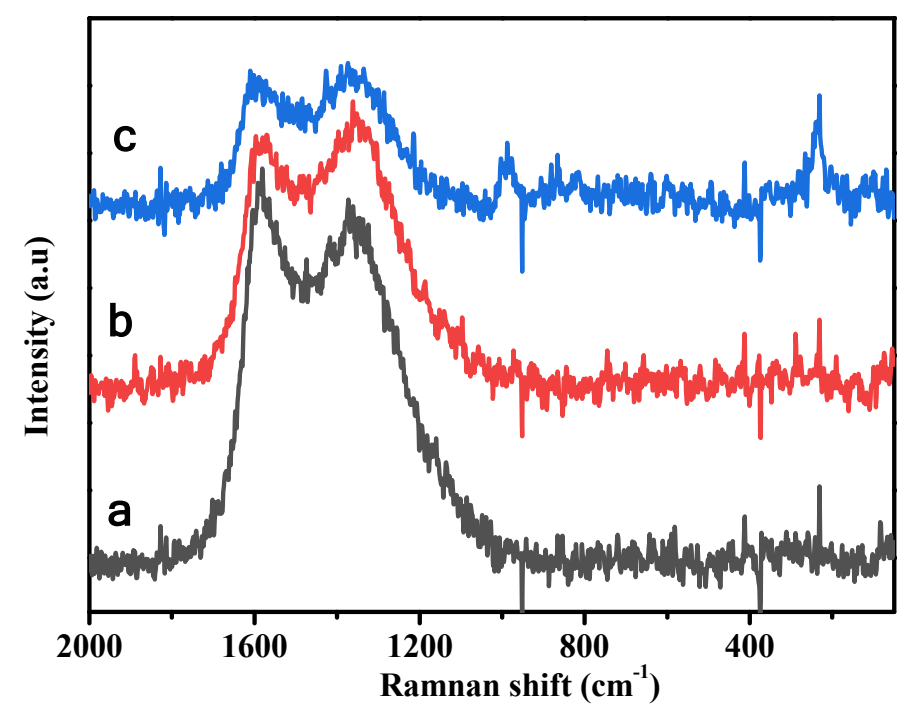

Figure S1. Raman spectra of (a) $\mathrm{MoO}_{x} / \mathrm{MC}-500$, (b) $\mathrm{MoO}_{x} / \mathrm{MC}-600$, (c) $\mathrm{MoO}_{x} / \mathrm{MC}-700$ 


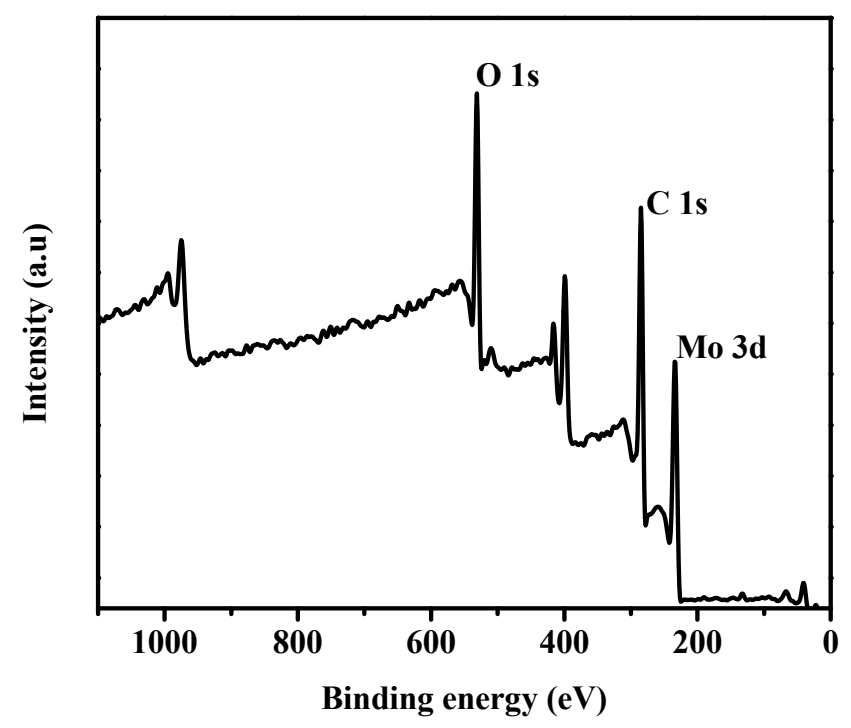

Figure S2. XPS spectra of $\mathrm{MoO}_{x} / \mathrm{MC}-600$

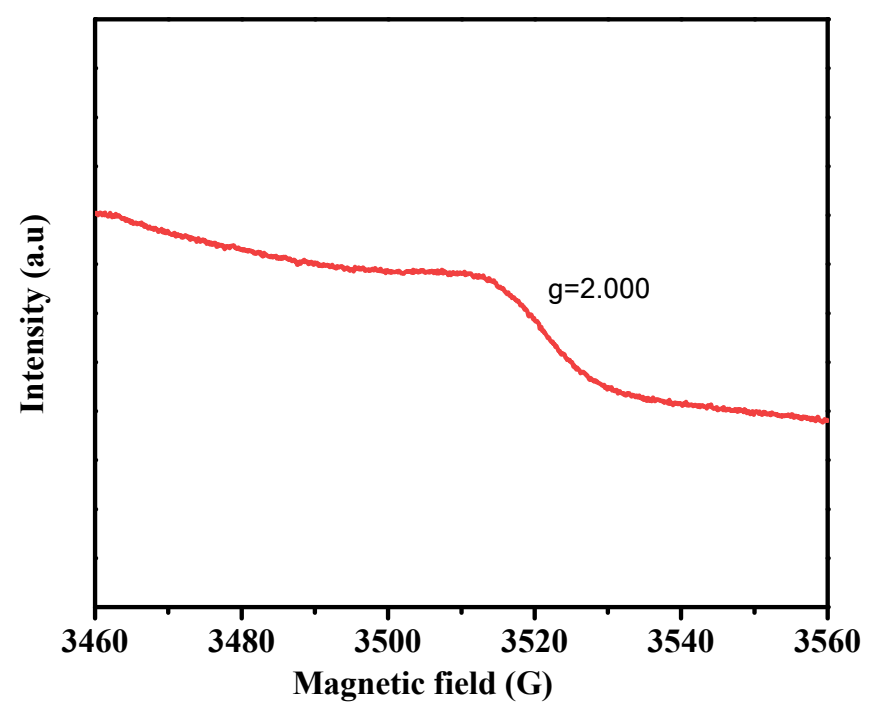

Figure S3. EPR spectra of $\mathrm{MoO}_{x} / \mathrm{MC}-600$ 


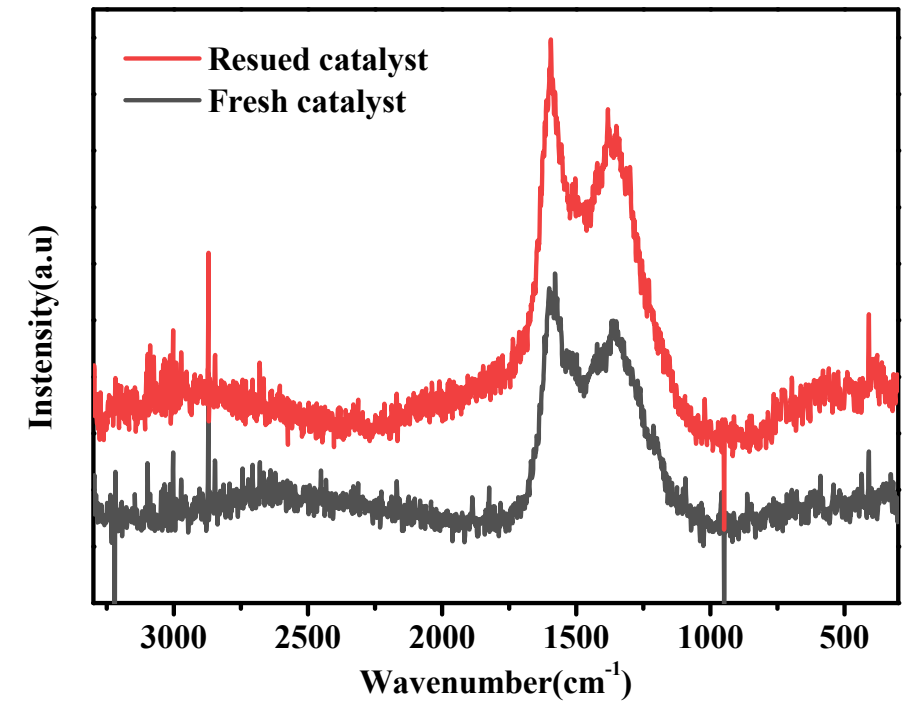

Figure S4 Raman spectrum of a) fresh catalyst and recycled catalyst 